\title{
JUEGO Y EDUCACIÓN CIUDADANA
}

\author{
Javier Monedero Gallego
}

\begin{abstract}
RESUMEN
Diversas disciplinas sociales han destacado el papel del juego en la sociedad como parte de la sublimación lúdica de la lucha por ganar y apropiarse de recursos. Los sicólogos evolutivos no definieron el juego sino que encontraron en él diversos momentos del proceso evolutivo de la mente y del carácter en la progresión cognitiva, el desarrollo moral y la maduración emotiva de la formación de los seres humanos. Este enfoque es básico para entender y aprovechar las posibilidades del juego como estrategia para la educación ciudadana, especialmente en la construcción e incorporación de las reglas del juego asociados a la convivencia y la ley.
\end{abstract}

Palabras clave: Juego - Educación ciudadana

\begin{abstract}
Pointed out differents socials disciplines the paper of the game in the society like part of the play sublimation of the fight for to win and to take of the resources. Evolution psychologists don't would define the game but that they found in it differents moments evolutionary process of the mind and the character in the cognitive progression, the moral development and the emotive maturation in the formation of humans living being. This focus is basic for understand and to take advantage the game possibilities like strategic for people education especially in the construction and incorporation the game's rules associated to living together and the law.
\end{abstract}

Key words: Play-People education

d

Qué tiene que ver el juego con los derechos humanos y con la ley, la competencia con la convivencia, el ensayo con el logro y todo lo anterior con la educación ciudadana?

Dicen los filósofos éticos modernos que la sociedad justa es la sociedad democrática regida bajo los principios universales de los derechos humanos que progresivamente ha ido construyendo la civilización actual. Se entiende que es un estado de conciencia social elevado que aspira a regirse por la justicia equitativa y la libertad de la decisión y la acción humana en una unidad dual entre individualidad y colectividad, diferencia e identidad. 
Los elementos del carácter que contribuyen a formar la decisión autónoma pero a la vez cooperativa, son hoy el sustento de los principios morales bajo los cuales se valora la actitud y la acción humanas, así como los criterios de valor desde donde se cimienta la ley: la responsabilidad personal y el bien común; hoy por hoy ejes de la democracia moderna, que como dijimos se sustentan en los derechos humanos.

\section{¿QUÉ TIENE QUE VER EL JUEGO CON TODO ESTO?}

El juego no sólo contribuye a formar las reglas que regulan la interacción de los individuos en los grupos, sino también los límites de las rivalidades o competencias. Todos los seres vivos compiten por los recursos de la subsistencia; el ser humano ha llevado esta competencia hasta la guerra con los seres de otras especies y al parecer más encarnizadamente con los de su misma especie. De hecho muchos juegos infantiles y de adultos son una simulación o la guerra misma. En efecto para los historiadores tanto el juego como competencia nacen de la guerra, una sublimación de ésta, una manera de lograr la gloria del más fuerte. Los torneos de los ejércitos de los aqueos organizados por Aquiles ("el de los pies ligeros") en honor de Patroclo, su entrañable amigo, muestran a la lucha, la carrera de carros, las carreras, el lanzamiento de la lanza etc., como las destrezas del soldado y de entre ellos del mejor. Una cosa los diferencia de la guerra: perder no implica morir, ganar tampoco matar, con una contada excepción. En el inicio de los juegos olímpicos era más glorioso ser un campeón atlético que un excelente guerrero. Llegar a ser hijo epónimo de una ciudad estado Griega se lo disputaban los artistas y los atletas. La historia, que fue de las guerras, sólo nos ha legado la fama de lo militar. Los eventos del circo romano nos llegan en sus peores épocas, cuando se deforma la figura del gladiador. Incluso es dudoso que los mayas mataran al que triunfaba en el juego de la pelota. Mucha propaganda adversa se recoge como historia. Incluso en las competencias deportivas de los soldados se enseñaba el principio ético de respetar al que se rinde ante la superioridad del otro. Tal vez porque un hombre rendido en un combate real era un esclavo, un botín de guerra, mucho más valioso que un occiso. Así incluso el juego militar antiguo nos lega un rudimento del Derecho Internacional Humanitario de hoy: el respeto por el combatiente desarmado y por su extensión a los civiles. Los niños mismos cuando juegan a la lucha le preguntan al otro: ise rinde?; y no es raro verlos entablando discusiones sobre las reglas de sus guerras imaginarias.

En su relación con las más diversas actividades humanas al juego se le atribuyen papeles diferentes. Victoria Camps, en su libro Virtudes Públicas ${ }^{1}$, dice que lo más importante en el juego y la política no es participar sino ganar. Una visión claramente competitiva y de poder, que enfatiza los aspectos territoriales y de dominio, o sea, desde el punto de vista de los fines materiales o emotivos. Muy por el contrario Gustav Bally en 1945 retoma teorías de 1896, como las de Karl Gross, que argumentan que "las manifestaciones motoras que no parecen perseguir inmediatamente una finalidad vital pueden considerarse como juegos", o de 1930: "la eliminación

\footnotetext{
${ }^{1}$ Camps, Victoria, Virtudes públicas, Madrid, Espasa Calpe, 1993.
} 
de los sentimientos hostiles y de miedo constituye el supuesto del juego"2. De ideas semejantes a las dos últimas se desprende que el juego es sólo lúdica, es decir juego del placer, del goce que no compromete lo vital, la existencia. Ambas visiones introducen una percepción exclusivamente adulta acerca del juego. Es normal que se haga, pues "el hombre, por el contrario -de los animales, juega durante toda la vida”. Es entonces muy común ver el juego como un negocio o una lucha o como placer. Como igual de adulta es la mirada de que los juegos entre niños son actividades para ocupar el tiempo, su distracción, un asunto espontáneo y sin un valor trascendente.

Para el enfoque de este artículo nos interesa contrastar la valoración de los historiadores y de los filósofos con la de los sicólogos y pedagogos que asociaron, como casi toda persona haciendo uso del sentido común, el juego con la infancia. Esto nos remite al tema de si el juego es una invención cultural de los adultos o si está asociado a los procesos evolutivos que experimenta el ser humano durante su desarrollo. Sólo que los pedagogos buscaron aplicarlo a la enseñanza como estrategia didáctica. Montessori, Claparede, Decroly le dieron mucha importancia al juego, la plástica y en fin todo lo que implicara la creatividad y la variedad como motivadores y recursos para la enseñanza y el aprendizaje. A la versión que el juego es una actividad propia del tiempo libre y carente de todo significado, seriedad y rigor, los pensadores y experimentadores de la educación valoraron el juego como un instrumento a través del cual el niño conoce, experimenta y se adapta al mundo. Para los psicólogos, que estudiaron las formas y estructuras de la mente que aprende, se trata de hacer eficiente el aprendizaje para que las personas tengan tiempo de sobra y disfruten de la vida (Skinner) o se trata de hacer disfrutable el aprendizaje mismo (Piaget). Esto para simplificar la discusión entre aprender como repetición o aprender como innovación o creación.

Piaget volvió la mirada hacia la importancia del juego en el proceso de desarrollo del niño y pudo relacionarlo con las etapas de evolución del pensamiento y del criterio moral. Mostró cómo el paso de las fases egocéntricas y asociales del niño a fases sociales y de integración social se expresan en los juegos que ellos desarrollan. De hecho en su minucioso estudio ${ }^{4}$ sobre la formación de la conciencia moral y la ley, demostró el proceso que ocurre en el tránsito de las relaciones unilaterales de sumisión heterónomas a las relaciones bilaterales u horizontales de cooperación autónomas. Una apreciación sobre la formación de la ley y la conciencia frente a ella.

Este descubrimiento sistemático produjo sus efectos pedagógicos e influyó en investigadores de la talla de Lawrence Kohlberg que profundizó a Piaget en la evolución y la estimulación de la evolución de la conciencia moral entre los sujetos. El trabajo de Kohlberg realizó seguimiento a sujetos desde la infancia por más de dieciocho años para establecer los cambios en su percepción y acción moral. Así que simplificó los mecanismos de evaluación para su socialización pedagógicas (ejercicios de dilemas morales) y su relación con los procesos evolutivos de los períodos de

${ }^{2}$ Bally, Gustav, El juego como expresión de la libertad (1945), Fondo de Cultura Económica, México, 1992.

${ }^{3}$ Idem, p. 59

${ }^{4}$ Piaget, Jean, El criterio moral en el niño, Ediciones Martínez Roca, S. A., Barcelona, 1987. 
pensamientos (operaciones) con los niveles (tres) relacionales y los estadios (seis) del juicio moral. Las limitaciones de su estudio lo llevaron a incluir otras variables como la incidencia del medio socio-cultural en la formación y conservación de los estadios de conciencia moral y su correspondencia con la acción o comportamiento moral. Hoy en día otro admiradores, pero a la vez críticos de la obra de Kohlberg inducen a replantear la omisión de lo emocional en la formación del juicio moral.

Estos aportes de Piaget y Kohlberg a la comprensión de la formación de la conciencia y el comportamiento moral son la base de casi todas las metodologías que sustentan una pedagogía para los derechos humanos. En esta relación de los juegos y la formación de la conciencia moral, estos psicólogos no definieron el juego en general sino que lo relacionaron con los diversos periodos de la evolución del organismo humano. De lo cual se concluye que no hay una definición para el juego sino que existen diversos tipos de juego según los periodos de desarrollo y el significado relacional de los mismos. Piaget distinguió cuatro tipos de juegos, que se pueden vincular con sus periodos de desarrollo cognitivo, que Kohlberg, a su vez, relacionó con los tres grandes niveles por los que escala el desarrollo moral en su proceso evolutivo: I. Juegos de Ejercicios (de 1 a 2 años, relacionados con el periodo Sensorio-motor, y el nivel (1) de desarrollo moral preconvencional, estadio 1), II. Juegos Simbólicos (2 a 7/8 años, relacionados con el subperiodo preoperatorio y con el nivel preconvencional, estadio 2), III. Juego de Reglas (de 67 a 11-12 años, relacionados con el subperiodo de operaciones concretas con el nivel (2) convencional, estadios 3 y 4) y IV. Juegos de Construcción (de 11-12 a 16 años relacionado con el periodo de las operaciones formales y con el nivel (3) posconvencional y estadios de desarrollo moral 5 y 6). No por ello un nuevo periodo y estadio elimina tipos de juegos de fases anteriores, antes bien las incorpora y recontextualiza.

Los juegos de ejercicio (sensorio-motores), se inician en los primeros años de vida y ocupan una gran parte del tiempo del niño centrado en su propio placer de autodecubrimiento, inicialmente sin ninguna finalidad hacia el mundo de afuera. De tal forma que al final del año y medio está empleando su cuerpo, a través de gestos, para explicar el contenido imperfecto de sus mensajes verbales que aún no domina. Aquí su desarrollo moral es absolutamente egocéntrico y sólo busca su satisfacción.

A través de los juegos simbólicos, el niño establece una nueva manera de relacionarse con el mundo; éstos se inician alrededor de los dos años. Ejerciendo destrezas en estos juegos teatrales, el niño crea una representación del mundo mediante la imitación de los objetos y acciones de la realidad, adaptándola a sus necesidades. Es una manera de resolver los conflictos que se le presentan con el mundo de los adultos. Aquí puede imaginar y vivir su propio mundo que escapa a la vida reglada desde afuera, que rígidamente se le impone y no comprende. Pero también, como explica Juan Delval: "Los símbolos que el niño utiliza pueden estar construidos por él, cobrar su significado dentro de la situación y en relación con la actividad, pero al tener una relación con el objeto que designan pueden ser fácilmente compartidos con otros niños. Un mismo objeto puede variar su significado al cambiar el juego y así una botella puede representar 
un muñeco y convertirse más tarde en un cohete" ${ }^{2}$. En este periodo los juegos de ejercicios se integran a los simbólicos, y el niño no sólo adapta el mundo a sus significados, sino que intenta colocarse en el lugar del otro, para descubrir sus motivos reglados y estrictos en el mundo adulto. Un primer aprendizaje en la resolución de conflictos, básicos en la formación ciudadana de los sujetos y el fomento de sus relaciones sociales con sus pares. Sin embargo, este lenguaje simbólico es un código cuasi hermético de los niños, que fácilmente socializan entre ellos y que constituye una especie de enigma para los adultos, pues los infantes lo emplean en su mundo especial y separado del extraño e inaccesible mundo de los adultos. En este estadio su desarrollo moral le induce acatar la norma que le impone la autoridad, identificándose con ella por miedo y conveniencia.

Cuando el niño se descentra de su "egocentrismo", acepta participar del juego reglado. Ahí recontextualiza, poco a poco, la representación relativamente arbitraria que se ha hecho del mundo (simbolismo) y empieza a desplazarse mejor en el orden convencional del lenguaje como código de comunicación racional delimitado y sintáctico, a la vez que se inserta en el orden social de los adultos al experimentar lo que es la norma y la regla (equivalentes a ley de los adultos) de los grupos de pares y elabora juicios morales acerca del bueno y lo malo para todos. Aquí pasa por diversos momentos de la experimentación y elaboración del juego. Primero lo hace de manera arbitraria, luego siguiendo estrictamente la tradición de la norma y reglas entregadas desde afuera por niños mayores. Aquí su desarrollo moral lo impele a ser agradable a la norma y al poder, acatando el orden externo porque hay que quedar bien.

Cuando ya es un niño mayor no empieza un juego sin antes no haber dejado sentadas las reglas del juego que acuerda con sus congéneres como condición previa antes de entregarse al esfuerzo, la competencia y el placer al mismo tiempo. Este es el primer paso participativo en la construcción de la ley. Aquí su desarrollo moral lo hace aceptar la norma porque es conveniente para el bien común y representa la igualdad y la justicia. No de otra manera se explica que en el "mundo libre" la industria de los juegos sea una de las más variadas y rentables en el comercio internacional; para ello se vale de todas las herramientas tecnológicas posibles. Algunos juegos modernos traen las reglas preestablecidas y entonces reproducen cierto nivel de heteronomía, obligando a los participantes a la ley preestablecida y al uso de la autonomía sólo en la toma de decisiones sobre el moverse adecuadamente en los marcos de lo permitido. Es el caso de Monopolio, los de Play Station y demás... Otros juegos dejan en libertad a sus protagonistas de concertar el alcance de algunas reglas, es el caso de ciertas dinámicas de grupo que fomentan la negociación.

En los juegos de construcción el adolescente ejerce la crítica y fomenta la negociación de las reglas del juego, de la lúdica y de la vida entre sus pares y en puja con los adultos. En ellos se intenta reconstruir e incluso transformar el mundo. Mediante muchos procedimientos el adolescente construye modelos teóricos y materiales, que van desde explicaciones racionales o hipotéticas hasta estructuras y mecanismos operativos. Aquí su desarrollo moral, que ya tiende

${ }^{5}$ Delval, Juan, Crecer y pensar, Paidós, $5^{a}$ reimpresión, 1997, España. 
a los estadios posconvencionales, le permite entender la norma como una construcción cultural en la cual puede intervenir y con la cual puede identificarse. Su desarrollo moral puede tentar el último estadio (según el modelo de Kohlberg) donde se permite observar la ley, más allá de principios normativos, a través de la ley interior, es decir, movido por el compromiso de la ética trascendente que piensa en toda la humanidad y en el lugar de cada ser humano. En este nivel de desarrollo se introducen pedagógicamente los juegos de simulación y de roles, donde se reconstruye la realidad lo más fielmente o se le modifica lo más sustentablemente posible. Muy útil para las áreas académicas que estudian la acción del ser humano en la sociedad y en la naturaleza. Muy útil para la práctica de la toma de decisiones, la participación creativa, la negociación de situaciones difíciles, y también para el aprendizaje de la tolerancia y el desarrollo de esa inteligencia que nos impulsa a comprender lo que se ve desde otro lugar, con otros intereses, otras pasiones y otros mundos históricos y culturales.

El tránsito de la heteronomía a la autonomía ocurre con los traumatismos propios de la acomodación social, emocional y cognitiva. Esto lleva a que sea la experiencia quien enseñe acerca de los vehículos de la cooperación y la reafirmación de las identidades individuales y grupales. El juego es pues una experiencia formativa por sí misma y lo será más en cuanto pertenezca a un currículo escolar intencionado que busque los logros condicionados a medios adecuados, antes que en la verbalización de ellos y la enunciación aleccionadora, a través de la vivencia de acciones sociales que ocurren en el juego elegido para fines pedagógicos. Una interacción donde no prevalece el fin sobre el medio pedagógico. El juego es en sí mismo un medio estratégico, un justificador de fines, una didáctica integral por que combina la dificultad con el goce, una manera de ejercitar competencias para la vida.

Piaget observaba el juego como una actividad propia del niño que revelaba, sin la intervención del adulto, el nivel de desarrollo cognitivo y social. Vygotski a su vez advertía: "al igual que no podemos considerar el placer como una características definitoria del juego, me parece que las teorías que ignoran el hecho de que el juego completa las necesidades del niño desembocan en una intelectualización pedante del juego" ". Abogaba por la estimulación de lo que él llamó zona de desarrollo próximo que es el movimiento de evolución entre una fase de desarrollo logrado y el próximo de desarrollo potencial. Ya que el niño nunca está en una fase estática; una vez ha conquistado todos los logros cognitivos y morales de esa fase ya está en movimiento hacia otra más avanzada. De ahí que en algunos infantes de repente se observen cambios cognitivos repentinos, cuando antes había atraso o dificultad, y en otros, una consecución progresiva de nuevos logros. Con esta apreciación le devolvía el papel al maestro y establecía que el aprendizaje no es una actividad totalmente autónoma del niño (como sugieren el constructivismo piagetiano) sino una actividad cooperada entre el niño y el adulto. El adulto acompaña y facilita el desarrollo de evolución del niño, y éste procede por imitación, recreación y creación. Es así como el juego en Vygotski deriva en una significación de herramienta pedagógica más que en un campo de

\footnotetext{
${ }^{6}$ Vygotski, Lev, El desarrollo de los procesos lógicos superiores, Grijalbo Mondadori, Barcelona, abril de 1996, p. 141.
} 
observación de los estadios, fases o niveles de desarrollo alcanzados y dominados autónomamente.

Para los derechos humanos todo juego reglado introduce al niño en el respeto y observancia de la ley. Es indudable que la introducción de reglas abre las puertas para la asimilación del derecho y cabe al pedagogo elegir el derecho que impera o promueve la sociedad real o ideal que él representa. El acto de jugar no hace sino abrir la llave para aprendizajes; la intención curricular es el cántaro o el lecho donde se vierte. El fin se corresponde con el vehículo. Cualquier juego de reglas, unos más que otros, pueden servir para simular o experimentar temas genéricos que tengan que ver con el derecho, en especial con los derechos humanos. Reflexionar sobre los juegos que se practican estimula el desarrollo moral y el crecimiento de la perspectiva ética de la vida.

Ahora bien el juego no sólo permite la enseñaza del derecho y de las costumbres de la tradición, también incorpora sus valores. Para la enseñanza de los derechos humanos es básico establecer los principios morales de las relaciones humanas basadas en la dignidad. El juego es un medio excelente para lograr que niños y adultos experimenten el respeto en todas sus manifestaciones, la igualdad, la equidad, la tolerancia, la libertad, la responsabilidad, la cooperación. La solidaridad, la amistad, el amor, y otro como la sinceridad, la honestidad... siempre y cuándo se sepa para qué se juega.

En los juegos de construcción también operan las reglas pero esta vez incorporando el componente cognitivo en alto grado. Juegos que permiten reelaborar o recrear sistemas, mecanismos, procesos, dinámicas del azar. En ellos caben los llamados juegos de simulación, el intercambio de roles, los dilemas morales, el feed-back, dinámicas comunicativas y cualquier otra técnica lúdica, que incluso incorpora la estética y sobre todo el lenguaje.

Sin embargo, es muy popular atribuirle al juego un papel exclusivamente lúdico, un lugar para perder el tiempo, un peligro que promueve el desorden al que no se arriesgan los contenidos y metodologías serias de la enseñanza. Vygotski es casi desconcertante y categórico al afirmar que

"el atributo esencial del juego es una regla que se ha convertido en deseo. La noción de Spinoza acerca de 'una idea que se ha convertido en deseo, un concepto que se ha transformado en pasión', encuentra su prototipo en el juego, que es el reino de la espontaneidad y la libertad. Respetar las reglas es una fuente de placer. La regla vence porque es el impulso más fuerte. Una regla de este tipo es una regla interna, una regla de auto-limitación y auto-determinación, como dice Piaget, y no una regla que el niño obedece como si se tratara de una ley física. En pocas palabras, el juego brinda al niño una nueva forma de deseos. Le enseña a desear relacionando sus deseos a un 'yo' ficticio, a su papel en el juego y sus reglas. De este modo, se realizan en el juego los mayores logros del niño, logros que mañana se convertirán en su nivel básico de acción realy moralidad".

\footnotetext{
${ }^{7}$ Vygotski, Lev. El desarrollo de los procesos lógicos superiores, Grijalbo Mondadori, Barcelona, abril de 1996, p. 152.
} 
De esta manera los investigadores que han estudiado al niño para entender al niño nos han dado claves para entender al ser humano. El hombre también descubre que un espacio para desplegar su libertad y su autodescubrimiento, para poner a prueba sus fortalezas y aliviar sus debilidades es el juego. Hoy son más las estrategias grupales con adultos que se basan en el juego como un acceso a las herméticas barreras que en nuestras estructuras mentales y del carácter bloquean el replanteamiento de maneras de ser que colisionan con el prójimo y con nosotros mismos (véase La teoría de los juegos para la economía y las matemáticas). El juego obra terapéuticamente preservando intacta nuestra dignidad, es una metáfora para asumir experimentalmente los rigores de una realidad que no siempre estamos dispuestos a observar desde el punto de vista de otros. También un medio para comprender el tratamiento adecuado de los conflictos de intereses y las maneras como los hombres se comportan en los equilibrios y desequilibrios de la competencia del mercado y de la vida. Una manera alegre e hipotética de hacer ciudadanía o de detectar sus crisis y carencias.

\section{REFERENCIAS BIBLIOGRÁFICAS}

VYGOTSKI, LEV. El desarrollo de los procesos lógicos superiores, Grijalbo Mondadori, Barcelona, abril de 1996. DELVAl, JUAN. Crecer y pensar, Paidós, $5^{a}$ reimpresión, 1997, España.

PIAGET, JEAN. El criterio moral en el niño, Ediciones Martínez Roca, S. A., Barcelona, 1987.

BALLY, GUSTAV. El juego como expresión de la libertad (1945), Fondo de Cultura Económica, México, 1992. CAMPS, VICTORIA. Virtudes públicas, Madrid, Espasa Calpe, 1993.

Nunes de Almeida, PAulo. Educación lúdica, San Pablo, Santafé de Bogotá, 1998.

CARRERAS, EIJO, ESTANY y OTROS. Cómo educar en valores, Narcea, Madrid, 1997.

BANY, MARY A., JOHNSON, LOIS, V. La dinámica de grupo en la educación, Aguilar, Madrid, 1973.

NEILL, A. S. Summerhill, Fondo de cultura Económica, México, 1986.

PIAGET, JEAN, LORENZ, KONRAD, ERIKSONM, ERIK H. y OTROS. Juego y desarrollo, Grijalbo, México D. F., 1982.

DEWEY, JOHn. Democracia y educación, Morata, Madrid, 1996.

MONEDERO, JAVIER. Eljuego y la participación, Red de formación pedagógica en educación para la democracia,

(Boletín No 1) Gobernación del Valle del Cauca, secretaría de Educación, 1997.

MORALES PUERTAS, MANUEL. "Un juego de rol para la cohesión”, Cuadernos de Pedagogía No 250, septiembre,

Barcelona, 1996.

ZUlLiger, HANS. Psicoterapia infantil por el juego, Ediciones Sígueme, Salamanca, España, 1968.

NAPIER, RODNEY W. y GERSHENFELD, MATTI K. Grupos: teoria y experiencia, Editorial Trillas, México, 1980.

Beal, George, Bohlen, joe M. y Raudabaugh, j. Neil. Conducción y acción dinámica del grupo, Editorial

Kapelusz, Buenos Aires, 1964.

RED de Solidaridad Social, Presidencia de la República. La solidaridad está en juego.

VILlEGAS, Víctor. 200 juegos y dinámicas, Ediciones Paulinas, Bogotá, 1987.

Caviedes, miguel. Dinámicas de grupo y relaciones humanas, Ediciones Palinas, Bogotá, 1994.

FLORIAN, SANDRA. Juegos ingeniosos para adolescentes, Coopertativa Editorial Magisterio, Bogotá, 2000.

Recibido en febrero 1 de 2004

Aprobado en junio 15 de 2004 